\title{
Effects of vertically- and horizontally-orientated plyometric training on physical performance: a meta-analytical comparison
}

Jason Moran ${ }^{\star 1}$

Rodrigo Ramirez-Campillo²

Bernard Liew ${ }^{1}$

Helmi Chaabene ${ }^{3,4}$

David Behm 5

Antonio García-Hermoso ${ }^{6,7}$

Mikel Izquierdo6, 8

Urs Granacher ${ }^{3}$

1. School of Sport, Rehabilitation and Exercise Sciences, University of Essex, Colchester, Essex, United Kingdom

2. Universidad de Los Lagos (University of Los Lagos), Department of Physical Activity Sciences, Research Nucleus in Health, Physical Activity and Sport, Laboratory of Measurement and Assessment in Sport, Osorno, Chile

3. Division of Training and Movement Science, University of Potsdam, Potsdam, Germany

4. High Institute of Sports and Physical Education, Kef, University of Jendouba, Jendouba, Tunisia

5. School of Human Kinetics and Recreation, Memorial University of Newfoundland, St. John's, Canada

6. Navarrabiomed, Complejo Hospitalario de Navarra (CHN), IdiSNA, Universidad Pública de Navarra (UPNA), Pamplona, Spain

7. Laboratorio de Ciencias de la Actividad Física, el Deporte y la Salud, Universidad de Santiago de Chile, USACH, Santiago, Chile

8. Grupo GICAEDS. Programa de Cultura, Física, Deporte y Recreación, UniversidadSanto Tomás, Bogotá, Colombia

Corresponding author contact details: jmorana@essex.ac.uk, +44 1452702482 


\section{ABSTRACT}

Background: In accordance with the principle of training specificity, adaptations to vertically- or horizontally-orientated plyometric training (VPT, HPT) directly transfer to athletic tasks that are carried out in the same direction as they are performed.

Objectives: The objective of this systematic review and meta-analysis was to determine the relative effect of VPT and HPT on both vertical and horizontal measures of physical performance.

Data sources: Google Scholar, CrossRef, Microsoft Academic, PubMed, Web of Science, Scopus.

Study eligibility criteria: To qualify for inclusion in the meta-analysis, studies must have included a plyometric training intervention that compared jumps executed in a vertical direction (i.e. countermovement jump [CMJ]) to jumps executed in a horizontal direction (i.e. standing horizontal jump).

Study appraisal and synthesis methods: We used the inverse-variance random effects model for meta-analyses. Effect sizes, calculated from measures of horizontally- or vertically-orientated performance, were represented by the standardised mean difference and presented alongside 95\% confidence intervals $(\mathrm{Cl})$.

Results: For between-group analysis on horizontal outcomes, there was a moderate, significant effect size (ES) in favour of HPT $(0.65$ [95\% Cl: $0.12,1.18], Z=2.41[\mathrm{p}=$ 0.02]). For the analysis on vertical outcomes, there was a trivial, non-significant difference between VPT and HPT $(-0.04[95 \% \mathrm{Cl}:-0.33,0.24], Z=0.0 .29[\mathrm{p}=0.77])$. Within-group analysis showed HPT to be superior to VPT across horizontally- $(1.05$ $[0.38,1.72]$ vs. $0.84[0.37,1.31])$ and vertically-orientated $(0.74[0.08,1.40]$ vs. $0.72[0.02$, 1.43]) performance measures. For horizontally-orientated outcomes, single-factor 
moderator analyses showed that longer programmes (>7 weeks), more sessions $(>12)$ and combined bilateral and unilateral training were most effective, favouring HPT in each case. In vertically-orientated outcomes, these same variables showed only trivial differences between HBT and VBT.

Conclusions: HPT is at least as effective as VPT at enhancing vertical performance but is superior at enhancing horizontal performance. This means that HPT might be a more efficient method for enhancing multi-vector performance for sport.

Key words: Vertical, horizontal, jump, exercise, stretch-shortening cycle

\section{Key points:}

- Horizontal plyometric training and vertical plyometric training are both effective for increasing jump and sprint performance.

- Horizontal plyometric training is at least as effective as vertical plyometric training at enhancing vertical performance but is superior at enhancing horizontal performance.

- A combination of bilateral and unilateral horizontal plyometric training seems to be the most efficient way to enhance jump and sprint performance. 


\section{Introduction}

The ability of the neuromuscular and musculoskeletal systems to generate force at a specific velocity, in a particular direction, appears to be critical for many sports that involve sprinting, jumping and throwing (1). In accordance with the principle of training specificity, sport-based demands, which require vertically- or horizontally-orientated force application, need to be addressed through training stimuli that possess these very same characteristics (2). For example, previous reviews $(3,4)$ outlined the importance of matching the characteristics of training stimuli to the demands of the sport being trained for. In this way, factors such as muscle action velocity and movement direction are considered to be key variables for coaches to manipulate (1). This is encapsulated by the concept of dynamic correspondence which is considered a robust theoretical framework for the formulation of sport-specific programming $(2,5)$.

The stretch and impact forces that occur during dynamic movement incite eccentric muscle actions with resultant elastic energy potentiating force production in subsequent concentric actions when coupling time is short $(6,7)$. This mechanism is an important factor in athletic performance with rapid movement underpinned by efficient usage of the stretch-shortening cycle (8). This has been argued to have implications for the programming of performance-maximising training in sport, with an appreciable body of literature supporting the principle of training specificity (2).

In light of the above, plyometric training (PT) can be used to enhance the ability of skeletal muscle to exert maximal force in as short a time as possible (9). In achieving this, an athlete can maximise their power output, resulting in improved performance in athletic tasks such as sprinting and jumping (10). This is an important physical ability 
given the time constraints associated with dynamic performance in sports such as soccer, basketball and tennis which, to some extent, can be mimicked in training.

Plyometric training typically includes various unilateral and bilateral jumps, hops and bounds $(9,11)$ with coaches often prescribing these in a multidirectional fashion to reflect the unpredictable nature of field and court sports. The rationale of this approach is founded on the well-accepted principle that adaptations to vertically- (VPT) and horizontally-orientated PT (HPT) will transfer better to athletic tasks that are carried out in the same direction as they are performed. For example, conventional thought suggests that a basketball player who jumps vertically to claim a rebound might, theoretically, benefit more from VPT such as CMJs; whereas a soccer goalkeeper who must rush towards an oncoming defender and quickly move laterally to block a shot on goal might, theoretically, benefit more from HPT such as horizontal jumps $(12,13)$.

The above assertions seem to be supported by randomised trials which have tested the theory of specificity with regard to the effect of plyometric training (PT) on physical performance. Dello lacono et al. (14) allocated study participants to one of two different drop jump training regimens. One group undertook VPT, whilst the other partook in HPT. Citing the effect of training specificity on the final results, the authors revealed that the execution of horizontal jumps lead to increases in sprinting and change of direction tests, whilst vertical jumps elicited greater increases in CMJ. The authors stressed the importance of using training methods that share common biomechanical characteristics with the task that they are designed to improve. In a similar study, Loturco et al. (10) assigned separate groups to either VPT or HPT, measuring the resultant effects on vertical and horizontal jumping tasks. Highlighting the importance of the relationship between training specificity and the "axis of movement", these researchers found that VPT had a relatively greater effect on a vertical jump test whilst 
HPT had a relatively greater effect on a horizontal jump test. Supportive research, in the form of principal component analysis, implies that jumping (vertical) and sprinting (horizontal) represent unique motor qualities that are underpinned by variable movement characteristics such as the speed of the stretch-shortening cycle (i.e. fast vs. slow) of a given action (15). The results of these studies might reflect the different force application strategies that are required to undertake different types of movement in different directions, thus underpinning the importance of the principle of specificity with regard to training stimuli.

In isolation, the above findings are both credible and logical, being supported by conventional biomechanical principles that are well-accepted in sport and exercise science. However, up until this point in time, no researcher has undertaken a pooled analysis of study results meaning the conclusions of individual studies only typically inform practitioners' approaches to PT programming. Accordingly, the objective of this systematic review and meta-analysis was to determine the effects of VPT and HPT on both vertical and horizontal measures of physical performance in athletic populations. The overall purpose was to better inform coaches' programming choices with regard to enhancing the specificity of the training stimulus.

\section{Methods}

This meta-analysis was conducted in accordance with the Preferred Reporting Items for Systematic Reviews and Meta-Analyses (PRISMA) statement (16).

\subsection{Literature search}

The Google Scholar, CrossRef, Microsoft Academic and PubMed databases were searched. With no date restrictions, a systematic search was first undertaken. Only articles published in the English language were considered. These searches were 
performed in March, 2020. The following search terms were used using the Boolean operator terms 'AND' and 'OR': 'ballistic', 'complex', 'explosive', 'force-velocity', 'plyometric', ‘stretch-shortening cycle', 'jump', 'plyometric exercise', 'resistance training', 'training', 'horizontal', 'vertical' (17). In selecting studies for inclusion, a review of all relevant article titles was conducted before an examination of article abstracts and, then, full published articles. Only peer-reviewed articles were included in the meta-analysis. After this systematic process, manual searches were also performed in authors' personal libraries, consisting of a large number $(n=7,859)$ of studies accumulated in a search initiated in April 2017. This search was updated in May, 2019 and remains ongoing with personal libraries receiving repeated updates on a weekly basis, through accounts created in different databases (Web of Science, Scopus and PubMed [including Medline]). Studies from authors' personal libraries were eligible for inclusion until the initiation of manuscript preparation in April, 2020.

\subsection{Data extraction}

Data were extracted from gathered articles with a form created in Microsoft Excel.

Where required data were not clearly or completely reported, article authors were contacted for clarification. In cases in which authors did not respond to our queries, their dataset was not considered for further analysis.

\subsection{Inclusion and exclusion criteria}

To determine the eligibility of studies for inclusion in this meta-analysis, we used the PICOS framework (16). These criteria are shown in Table 1 and the characteristics of the study participants are displayed in Tables 2 and 3.

Table 1 PICOS framework for study inclusion and exclusion criteria 
Table 2 Characteristics of study participants for horizontally-orientated training groups

Table 3 Characteristics of study participants for vertically-orientated training groups

\subsection{Analysis and interpretation of results}

Meta-analytical comparisons were carried out in RevMan version 5.3 (25). Means and standard deviations for measures of horizontal and/or vertical performance were used to calculate an effect size. The inverse-variance random effects model for metaanalyses was used because it allocates a proportionate weight to trials based on the size of their individual standard errors (26) and facilitates analysis whilst accounting for heterogeneity across studies (27). Effect sizes are represented by the standardised mean difference and are presented alongside 95\% confidence intervals $(\mathrm{Cl})$. The calculated effect sizes were interpreted using the conventions outlined for standardised mean difference by Hopkins et al $(28)(<0.2=$ trivial; 0.2-0.6 = small, 0.6 $1.2=$ moderate, $1.2-2.0$ = large, 2.0-4.0 = very large, $>4.0=$ extremely large $).$ In cases in which there was more than one intervention group in a given study, the comparison group was proportionately divided to facilitate comparison across all participants (29).

To gauge the degree of heterogeneity amongst the included studies, the $R$ statistic was calculated. This represents the proportion of effects that are due to heterogeneity as opposed to chance (16). Low, moderate and high levels of heterogeneity correspond to $R$ values of $25 \%, 50 \%$ and $75 \%$ respectively; however, these thresholds are considered tentative (30). The $X^{2}$ (chi square) assesses if any observed differences in results are compatible with chance alone. A low $\mathrm{P}$ value, or a large chi- 
squared statistic relative to its degree of freedom, provides evidence of heterogeneity of intervention effects beyond those attributed to chance (26).

\subsection{Assessment of risk of bias}

The Physiotherapy Evidence Database (PEDro) scale was used to assess the risk of bias and methodological quality of eligible studies included in the meta-analysis. This scale evaluates internal study validity on a scale from 0 (high risk of bias) to 10 (low risk of bias). Two reviewers (JM, AGH) independently rated each study in accordance with the PEDro scale. Any ratings that yielded different results were further adjudicated by a third reviewer (UG). This rating was then used in the risk of bias scale. A median score of $\geq 6$ represents the threshold for studies with low risk of bias (31).

\subsection{Analysis of moderator variables}

To assess the potential effects of moderator variables, subgroup analyses were performed for both horizontally- and vertically-orientated outcome measures. Using a random effects model, we selected potential moderators likely to influence the effects of training. This included the number of weeks in the applied programme, the total number of training sessions in the programme (8), the bilateral/unilateral classification of the type of HPT or VPT performed (32), the training status of the study participants (33) and the chronological age of the study participants (8). For number of weeks and total sessions, a median split was used to form the subgroups. For jump type classification, the effects of bilateral PT were compared to bilateral combined with unilateral as very few studies examined the effects of unilateral only. For training status, those cohorts whom study authors reported as 'untrained' comprised one subgroup whilst the other was formed by individuals with no less than three years of 
training experience. For age, subgroups were divided into $\leq 18$ years and $>18$ years groups.

\section{Results}

\subsection{Study selection}

In total, nine studies met the inclusion criteria and were included in the systematic review. The PRISMA flow diagram illustrating the number of studies excluded at each stage of the systematic review and meta-analysis is shown in Figure 1. Together, the studies achieved the required standard to be considered to be at a low risk of bias (median quality score $=6.0$ ). These data are presented in Table 4.

\section{Figure 1 Flow chart for inclusion and exclusion of studies}

\section{Table 4 Results of risk of bias analysis using the PEDro scale}

\subsection{Primary analyses}

Two primary analyses were undertaken in this study. In each case, HPT was compared to VPT with the first analysis relating to horizontally-orientated outcome measures (i.e. horizontal jump, sprints) and the second pertaining to verticallyorientated outcome measures (i.e. CMJ). Nine studies were included in this metaanalysis. The vertical outcome analysis included ten experimental groups and the horizontal analysis included eight. For the analysis on horizontal outcomes, there was a moderate, significant effect size (ES) in favour of HPT $(0.65[0.12,1.18], Z=2.41[p$ $=0.02])$. Between-study heterogeneity was moderate and significant $(R=62 \%[p=$ 0.009]). These results are displayed in Figure 2. For the analysis on vertical outcomes, there was a trivial, non-significant difference between VPT and HPT -0.04 [-0.33, 
$0.24]], Z=0.29[p=0.77])$. Between-study heterogeneity was low and non-significant $(R=0 \%[p=0.56])$. These results are displayed in Figure 3.

Figure 2 Forest plot of comparison of horizontally- vs vertically-orientated plyometric training on horizontally-orientated measures of performance

Figure 3 Forest plot of comparison of horizontally- vs vertically-orientated plyometric training on vertically-orientated measures of performance

\subsection{Within-group effects of intervention}

We also explored the within-group intervention effects of each PT protocol. In this analysis, the effects of HPT on both horizontally- and vertically-orientated performance outcomes, as well as the effects of VPT on both horizontally- and vertically-orientated performance outcomes were determined. These results are displayed in Table 5.

Table 5 Effect sizes and $95 \%$ confidence intervals for within-group performance of horizontally- and vertically-orientated outcomes

\subsection{Effect of moderator variables}

The results of the moderator analysis are displayed in Tables 6 and 7. For both horizontally- and vertically-orientated outcome moderators, differences between subgroups were non-significant with low to moderate heterogeneity. For horizontallyorientated outcomes, the effect size for programme duration, as measured by number of weeks, was larger for those studies longer than seven weeks $(0.96[-0.15,2.08])$ than it was for those shorter than 7 weeks $(0.43[-0.00,0.86])$ and favoured HPT in both cases. For vertically-orientated outcomes, there was little difference between longer (>8 weeks; $-0.19[-0.83,0.46])$ and shorter programmes $(\leq 8$ weeks; $0.08[-0.28$, 0.44]. For horizontally-orientated outcomes, there was a large effect size (1.91 [0.87, 
2.96]) for programmes that had more than twelve sessions, with only a small effect size $(0.30[-0.06,0.66])$ in those that had fewer than twelve. Again, this favoured HPT in both cases. The trend was not apparent for vertically-orientated outcomes with only trivial differences between VPT and HPT for programmes with greater (>12 sessions; $-0.19[-0.83,0.46])$ or fewer (<12 sessions; $0.08[-0.28,0.44])$ sessions. For horizontally-orientated outcomes, a combination or bilateral and unilateral PT was more effective $(0.98[0.23,1.73])$ than bilateral only $(0.18[-0.32,0.69])$, with HPT favoured over VPT. For vertically-orientated outcomes, there was little difference between these types of PT with trivial differences apparent. It was only possible to conduct a moderator analysis for training status for vertically-orientated measures. Here, we found no difference between the effects of HPT and VPT in trained participants $(-0.15[-0.47,0.18])$ with HPT being slightly more effective for untrained participants $(0.29[-0.29,0.87])$. For chronological age, in horizontal measures, both age groups (>18 years; $\leq 18$ years) demonstrated moderate effects $(0.65[0.00,1.30]$ vs. $0.70[-0.27,1.68])$ whilst for vertical measures, both groups demonstrated trivial effects $(-0.09[-0.52,0.34]$ vs. $0.00[-0.39,0.39])$. For the horizontal outcome measures, HPT was favoured, regardless of age group.

\section{Table 6 Moderator analyses for horizontally-orientated outcome measures}

\section{Table 7 Moderator analyses for vertically-orientated outcome measures}

\section{Discussion}

\subsection{Main findings}

The purpose of this systematic review and meta-analysis was to determine the effect of VPT and HPT on both vertical and horizontal measures of physical performance in athletic populations. In sport and exercise science, the principle of training specificity 
supports the use of vertical and horizontal jumps to better improve performance in vertically- and horizontal-orientated tasks respectively (34). Accordingly, the principle of training specificity informs the type of training stimuli that a coach chooses for an athlete to optimise performance. In this way, a horizontal jump might be more effective than a vertical jump in eliciting increases in sprint speed, whereas a vertical jump may be more beneficial for a sports skill such as a rebound jump in basketball. However, the results of this meta-analysis seem to suggest that this principle may not be as clear as this and that other factors could play a role in the relative effectiveness of HPT and VPT. Our results suggest that HPT is at least as effective as VPT at enhancing vertical performance but is superior at enhancing horizontal performance.

Recently, there has been an increased interest in fine-tuning the specificity of training stimuli to meet the demands posed within sport. For example, it has been argued that force production in a horizontal direction is vital in underpinning acceleration capacity in athletes with horizontally-orientated weighted sled pushing thought to be an appropriate method to training this physical capacity (35). This interest has been generated by research which has highlighted the shortcomings of vertically-orientated exercise for the enhancement of horizontally-orientated movement. For instance, it has previously been shown that that a $10 \%$ increase in squat strength did not result in any change to $30 \mathrm{~m}$ sprint performance over a 9 week period $(3,36)$. Similarly, a previous review indicated that significant increases is sprinting speed were commonly unaccompanied by increases in vertically-orientated strength (37). In contrast, Morin et al. (35) found that heavy sled pushing resulted in a $1.2 \%$ increase in $20 \mathrm{~m}$ sprint speed over a shorter time period. In recreational athletes at least, speed increases of this magnitude are thought to be stimulated by strength increases of around $23 \%(37)$, potentially indicating a minimum threshold for improvement. However, this may differ 
in horizontal and vertical directions. Through three-dimensional modelling of both horizontal and vertical jumps, Nagano et al. (38) elucidated the differing characteristics inherent to these respective movement patterns. The researchers, examining the horizontal and vertical positioning of the centre of mass during take-off, demonstrated different positions of the body's centre of mass in both horizontal and vertical jumps. Vertical jumps showed practically no displacement of the centre of mass in a horizontal direction at take-off. However, the difference in the vertical displacement of the centre of mass was comparable between both types of jump. This implies that there is a horizontal and vertical component to horizontal jumps, whereas verticaljumps possess a vertical component only. Cappa and Behm (39) compared drop (vertical) and hurdle (vertical and horizontal) jumps finding shorter ground contact times, higher vertical ground reaction forces, greater limb stiffness and generally higher muscle activation in the lower limbs (rectus femoris, biceps femoris and gastrocnemius) with the horizontally-orientated hurdle jumps. The subsequent influence on the application and direction of ground reaction forces in both horizontal and vertical jumps seems to be evident in the results seen in the current meta-analysis and is likely to be the reason for the pattern of adaptation that we report.

Our results could also be explained by the type of performance tests assessed in this meta-analysis. For the vertical analysis only jumps were assessed, however for the horizontal analysis, both jumps and short sprints were used. Loturco et al. (10) found that whilst horizontally-orientated training was effective in enhancing speed and acceleration capacities over short distances (i.e. $<10 \mathrm{~m}$ ), vertically-orientated training resulted in greater performance improvements over longer distances, yet there were no tests of this type in this meta-analysis. This could have put VPT at a disadvantage to HPT when it came to assessing the effect of interventions on the included 
dependent variables. Loturco et al. (10) argued that this was due to the increasingly important role of vertical ground reaction forces at higher velocities over longer distances. This is reinforced by previous research (40) which demonstrates relatively larger vertical ground reaction forces in the latter stages of a $50 \mathrm{~m}$ sprint. This is concurrent to a progressively lower contribution of anterior/posterior ground reaction forces (40). In this way, though HPT seems the more effective and efficient training method based on the current meta-analysis, vertical jumps can still play an important role in the programmes of athletes who must sprint over longer distances (i.e. $>40 \mathrm{~m}$ ).

As expected, HPT was more effective in enhancing horizontal performance but it was no less effective than VPT at enhancing vertical performance. A key pattern of our results means that the opposite cannot be claimed and this seems to render HPT more important for sports which are characterised by extensive horizontal and vertical locomotion. Aside from the obvious efficacy of HPT to enhance direction-specific performance, these findings could provide efficiency benefits for coaches and athletes alike. For example, congested training and playing schedules are common in many sports, causing excessive physical stress which, in turn, can result in burnout or injury (41). Previous evidence indicates that athletes experience negative outcomes relating to physical performance and hormonal profile (42) during such periods of higher density training (43), and this necessitates the careful balancing of workloads to preserve optimal condition. In support of this, it is important to note that lower volumes of PT have provided greater training benefits than higher volumes when integrated with balance $(44,45)$ and other forms of resistance training $(46)$. Based on our results, the preferential use of HPT over VPT might be able to facilitate this balance by potentially giving coaches the freedom to programme just one directional form of PT, for example HPT, with the understanding that both vertical and horizontal performance 
could be optimised and maintained. This seems to be facilitated by the aforementioned characteristics of HPT which possesses both vertical and horizontal components compared to the singular vertical component of VPT (38). The preferential use of HPT would therefore seem to represent a more elegant and time-efficient form of programming that provides both performance and scheduling benefits which could offset strain on an athlete whilst optimising performance.

\subsection{Moderating variables}

Moderator analysis was undertaken for both horizontally- and vertically-orientated outcome measures. Aside from highlighting potentially important moderators of the main effects seen in this meta-analysis, the evaluation of these moderators could also have implications for the prescription of both HPT and VPT in athletic populations. Unsurprisingly, the moderator analysis supported the use of longer programme $(>7$ weeks) and more training sessions per programme $(>12)$ for the enhancement of horizontally-orientated outcomes and skills such as horizontal jumps and short sprints. In this case, HPT was favoured over VPT. In vertically-orientated outcomes, these same variables (>8 weeks, >14 session) displayed only trivial differences between subgroups, with neither HPT or VPT favoured. This would seem to reinforce the main effects but could also indicate a differential in the time-course of adaptation of vertically- and horizontally-orientated performance, or a potential bias towards the selection of vertically-orientated exercises in modern strength and conditioning programmes for athletes (47). On this, it is not inconceivable that many athletes already possess a high level of VPT experience alongside a concurrent lower level of expertise in relation to HPT. This could lower the ceiling of potential future adaptation (33) in vertically-orientated tests and this pattern was seemingly apparent in the results we found in the moderator analysis for training status. If this is the case, a transition 
by coaches to more horizontally-orientated exercises and outcome measures is warranted, though not at the complete expense of vertically-orientated. Had we been able to carry out a moderator analysis for training status for the horizontal measures of performance, we may have been able to provide greater clarity on this particular finding.

Another notable result from the moderator analysis for horizontally-orientated outcome measures was the finding that a combination of bilateral and unilateral PT was substantially more effective than bilateral PT executed in isolation. Reflecting the previous results, the positive effect sizes indicated that HPT was the favourable method with which to achieve these performance increases, with no difference between VPT or HPT for the vertical moderators. Recent research from Bogdanis et al. (48) found that unilateral PT was superior to bilateral PT at enhancing both unilateral and bilateral CMJ performance. The authors speculated that neural factors played a role in this pattern of adaptation, highlighting that unilateral training has previously been shown to result in greater electromyographic activity in the vastus medialis and gastrocnemius muscles (49). This effect seems to be further enhanced by the greater impulses developed during unilateral PT, due primarily to the manifestation of the bilateral deficit and the longer muscle action times associated with this type of jump (50). Chronological age was used as a proxy variable to denote maturation status as in a previous meta-analysis (8). We divided the subgroups into those older and younger than 18 years on the basis that maturational changes during childhood and adolescence, as well as training age, can result in both increases and decreases in performance due to a number of dynamic factors (8). For vertical outcome measures, there were only trivial differences between HPT and VPT in both age-related subgroups whilst for horizontal outcomes, each method yielded moderate 
effect sizes in both age divisions, favouring HPT. These results once again indicate the superiority of HPT over VPT in eliciting sport-specific performance increases with no differences observed between individuals older or younger than 18 years. In this way, similar principles could apply to the programming of both VPT and HPT across age groups with performance increases of similar a magnitude expected regardless of age profile. However, coaches must ensure that technique and technical competency are present regardless of the age of the athlete and so that particular requirement should be a guide to where an individual resides on the continuum of expertise as it relates to $\mathrm{PT}$. On this factor, coaches should also consider the relative movement challenges posed by individual jump types; for example, it may be relatively easier to perform a vertical jump than a horizontal jump, or a bilateral jump than a unilateral jump. On this basis, regardless of the apparent superiority of HPT over VPT, coaches should choose those exercises that an individual is physically capable of executing, adding layers of complexity as skill proficiency is attained.

\subsection{Future research}

Some studies in this meta-analysis did include interventions that examined the effect of combined HPT and VPT on physical performance but not enough investigations of this type exist to come to clear conclusions on the efficacy of a blended approach to programming. It could, therefore, be that whereas HPT seems superior to VPT for the enhancement of physical performance in isolation, a combination of the two could be optimal. This has previously been demonstrated (24) but further investigation in this area could reveal whether or not combined directional PT could be superior to singular HPT or VPT. Studies must also be carried out in female populations. Not a single study in this meta-analysis was conducted in female participants meaning its applicability to that population is undermined. This needs urgent addressing as females' jump 
landings are characterised by increased knee valgus and higher vertical ground reaction forces, compared to males (51). This could have a negative impact on adaptations to VPT and HPT alike.

\subsection{Limitations}

There are some limitations to the current study so our results should be interpreted with caution. For subgroup analyses, the dichotomisation of continuous data with median split could result in residual confounding and reduced statistical power $(52,53)$. Furthermore, moderator analyses were calculated independently, and not interdependently. Such univariate analysis must be interpreted with caution because the programming parameters were calculated as single factors, irrespective of between-parameter interactions. Also, as in many meta-analyses, we observed varying levels (low to high) of heterogeneity between trials meaning that some of these results must be viewed with caution. More studies and better standardisation of methods across trials could address this issue in future work.

\section{Conclusion}

Whilst HPT and VPT are both effective at increasing performance in horizontal and vertical directions respectively, HPT is at least as effective as vertical plyometric training at enhancing vertical performance but is superior at enhancing horizontal performance. Based on these results it seems logical to recommend that coaches include a higher proportion of HPT in athletes' programmes, particularly if their sport requires an extensive horizontal component. Coaches should still aim to include both types of jump in athletes' programmes as research has shown that each display unique biomechanical characteristics and applications to different elements of physical performance. However, in time-constrained scenarios, the use of HPT only strategies 
could be advantageous in optimising performance and maintaining an athlete's condition. Overall this means that HPT might be a more efficient method for enhancing multi-vector performance in athletes. Further to this, it seems that the positive effects of HPT can be enhanced through the execution of a combination of bilateral and unilateral HPT, reflecting the multidimensional nature of sport and leveraging the bilateral deficit to ensure maximal adaptation.

\section{Data sharing statement}

There are no underlying data this should be stated.

\section{Compliance with Ethical Standards}

Funding

No sources of funding were used to assist in the preparation of this article.

\section{Conflicts of Interest}

Jason Moran, Rodrigo Ramirez-Campillo, Bernard Liew, Helmi Chaabene, David Behm, Antonio García-Hermoso, Mikel Izquierdo and Urs Granacher declare that they have no conflicts of interest relevant to the content of this review.

\section{Authorship Contributions}

$\mathrm{JM}$ collected the data, analysed the data and wrote the manuscript, RRC collected the data and wrote the manuscript, $\mathrm{BL}$ analysed the data and wrote the manuscript, $\mathrm{HC}$ analysed the data and wrote the manuscript, DB wrote the manuscript, $\mathrm{AGH}$ analysed the data and wrote the manuscript, MI wrote the manuscript, UG analysed the data and wrote the manuscript. 


\section{References}

1. Izquierdo M, Häkkinen K, Gonzalez-Badillo JJ, Ibáñez J, Gorostiaga EM. Effects of long-term training specificity on maximal strength and power of the upper and lower extremities in athletes from different sports. Eur J Appl Physiol. 2002;87(3):264-71.

2. Goodwin JE, Cleather DJ. The biomechanical principles underpinning strength and conditioning. In: Strength and conditioning for sports performance. New York: Routledge; 2016.

3. Young WB. Transfer of strength and power training to sports performance. Int J Sports Physiol Perform. 2006;1(2):74-83.

4. Behm DG, Sale DG. Velocity specificity of resistance training. Sport Med Eval Res Exerc Sci Sport Med. 1993;15(6):374-88.

5. Fitzpatrick DA, Cimadoro G, Cleather DJ. The magical horizontal force muscle? A preliminary study examining the "force-vector" theory. Sports. $2019 ; 7(2): 30-8$.

6. Nicol C, Avela J, Komi P V. The stretch-shortening cycle. Sports Med. $2006 ; 36(11): 977-99$.

7. Komi P. Stretch-shortening cycle. Strength and Power in Sport. Chichester: John Wiley \& Sons; 2008.

8. Moran J, Sandercock GRH, Ramírez-Campillo R, Meylan C, Collison J, Parry DA. Age-related variation in male youth athletes' countermovement jump following plyometric training. J Strength Cond Res. 2017;31(2):552-65.

9. Chu D, Myer G. Plyometrics. Champaign: Human Kinetics; 2013. 
10. Loturco I, Pereira LA, Kobal R, Zanetti V, Kitamura K, Abad CCC, et al. Transference effect of vertical and horizontal plyometrics on sprint performance of high-level U-20 soccer players. J Sports Sci. 2015;33(20):2182-91.

11. Ramirez-Campillo R, Álvarez C, García-Hermoso A, Ramírez-Vélez R, Gentil P, Asadi A, et al. Methodological characteristics and future directions for plyometric jump training research: a scoping review. Sports Med. 2018;48(5):1059-81.

12. Cappa DF, Behm DG. Training specificity of hurdle vs. countermovement jump training. J Strength Cond Res. 2011;25(10):2715-20.

13. Ziv G, Lidor R. Vertical jump in female and male basketball players: a review of observational and experimental studies. J Sci Med Sport. 2010;13(3):332-9.

14. Iacono A Dello, Martone D, Milic M, Padulo J. Vertical- vs. horizontal-oriented drop jump training: chronic effects on explosive performances of elite handball players. J Strength Cond Res. 2017;31(4):921-31.

15. Salaj S, Markovic G. Specificity of jumping, sprinting, and quick change-ofdirection motor abilities. J Strength Cond Res. 2011;25(5):1249-55.

16. Liberati A, Altman DG, Tetzlaff J, Mulrow C, Gøtzsche PC, loannidis JPAA, et al. The PRISMA statement for reporting systematic reviews and meta-analyses of studies that evaluate health care interventions: explanation and elaboration. J Clin Epidemiol. 2009;62(10):e1-34.

17. Ramirez-Campillo R, Moran J, Chaabene H, Granacher U, Behm DG, GarcíaHermoso A, et al. Methodological characteristics and future directions for 
plyometric jump training research: A scoping review update. Scand J Med Sci Sport. 2020;30(6):983-97.

18. Abass A. Correlational Effects Of Plyometric Training On Leg Muscle Strength, Endurance And Power Characteristics Of Nigerian University Undergraduates. Int J African African Am Stud. 2005;4(1):42-52.

19. Gonzalo-Skok O, Sánchez-Sabaté J, Izquierdo-Lupón L, Sáez de Villarreal E. Influence of force-vector and force application plyometric training in young elite basketball players. Eur J Sport Sci. 2019;19(3):305-14.

20. Hortobagyi T, Havasi J, Varga Z. Comparison of two stretch-shortening exercise programmes in 13-year-old boys-nonspecific training effects. J Hum Mov Stud. 1990;18:177-88.

21. Keller S, Koob A, Corak D, von Schöning V, Born DP. How to Improve Change-of-Direction Speed in Junior Team Sport Athletes-Horizontal, Vertical, Maximal, or Explosive Strength Training? J strength Cond Res. $2020 ; 34(2): 473-82$.

22. Arcos AL, Yanci J, Mendiguchia J, Salinero JJ, Brughelli M, Castagna C. Short-term training effects of vertically and horizontally oriented exercises on neuromuscular performance in professional soccer players. Int J Sports Physiol Perform. 2014;9(3):480-8.

23. Manouras N, Papanikolaou Z, Karatrantou K, Kouvarakis P, Gerodimos V. The efficacy of vertical vs. Horizontal plyometric training on speed, jumping performance and agility in soccer players. Int J Sport Sci Coach. $2016 ; 11(5): 702-9$. 
24. Ramirez-Campillo R, Gallardo F, Henriquez-Olguin C, Meylan CMP, Martinez C, Alvarez C, et al. Effect of vertical, horizontal, and combined plyometric training on explosive, balance and endurance performance of young soccer players. J Strength Cond Res. 2015;29(7):1784-95.

25. The Nordic Cochrane Centre. Review Manager. Cochrane Collaboration. 2014. p. 1-43.

26. Deeks JJ, Higgins JP, Altman DG. Analysing data and undertaking metaanalyses. In: Cochrane Handbook for Systematic Reviews of Interventions: Cochrane Book Series. 2008. p. 243-96.

27. Kontopantelis E, Springate DA, Reeves D. A re-analysis of the Cochrane Library data: the dangers of unobserved heterogeneity in meta-analyses. PLoS One. $2013 ; 8(7)$.

28. Hopkins WG, Marshall SW, Batterham AM, Hanin J. Progressive statistics for studies in sports medicine and exercise science. Med Sci Sports Exerc. $2009 ; 41(1): 3-12$.

29. Higgins JP, Deeks JJ, Altman DG. Special Topics in Statistics. In: Cochrane Handbook for Systematic Reviews of Interventions: Cochrane Book Series. 2008. p. $481-529$.

30. Higgins JPT, Thompson SG, Deeks JJ, Altman DG. Measuring inconsistency in meta-analyses. BMJ Br Med J. 2003;327(7414):557-60.

31. Maher CG, Sherrington C, Herbert RD, Moseley AM, Elkins M. Reliability of the PEDro scale for rating quality of randomized controlled trials. Phys Ther. 2003;83(8):713-21. 
32. Bobbert MF. Explanation of the bilateral deficit in human vertical squat jumping. J Appl Physiol. 2006;100(2):493-9.

33. Hawley JA. Specificity of training adaptation: time for a rethink? J Physiol. $2008 ; 586(1): 1-2$.

34. Randell AD, Cronin JB, Keogh JWL, Gill ND. Transference of strength and power adaptation to sports performance-horizontal and vertical force production. Strength Cond J. 2010;32(4):1524-602.

35. Morin JB, Petrakos G, Jiménez-Reyes P, Brown SR, Samozino P, Cross MR. Very-heavy sled training for improving horizontal-force output in soccer players. Int J Sports Physiol Perform. 2017;12(6):840-4.

36. Harris GR, Stone MH, O’Bryant HS, Proulx CM, Johnson RL. Short-term performance effects of high power, high force, or combined weight-training methods. J Strength Cond Res. 2000;14(1):14-20.

37. Cronin J, Ogden T, Lawton T, Brughelli M. Does increasing maximal strength improve sprint running performance? Strength Cond J. 2007;29(3):86-95.

38. Nagano A, Komura T, Fukashiro S. Optimal coordination of maximal-effort horizontal and vertical jump motions - A computer simulation study. Biomed Eng Online. 2007;6(1):20-9.

39. Cappa DF, Behm DG. Neuromuscular characteristics of drop and hurdle jumps with different types of landings. J Strength Cond Res. 2013;27(11):3011-20.

40. Nagahara R, Mizutani M, Matsuo A, Kanehisa H, Fukunaga T. Association of sprint performance with ground reaction forces during acceleration and maximal speed phases in a single sprint. J Appl Biomech. 2018;34(2):1-20. 
41. Soligard T, Schwellnus M, Alonso JM, Bahr R, Clarsen B, Dijkstra HP, et al. How much is too much? (Part 1) International Olympic Committee consensus statement on load in sport and risk of injury. Br J Sports Med. 2016;50(17):1030-41.

42. Jones CM, Griffiths PC, Mellalieu SD. Training load and fatigue marker associations with injury and illness: a systematic review of longitudinal studies. Sports Med. 2017;47(5):943-74.

43. Sanchez-Sanchez J, Sanchez M, Hernandez D, Ramirez-Campillo R, Martínez C, Nakamura FY. Fatigue in U12 soccer-7 players during repeated one-day tournament games - a pilot study. J Strength Cond Res. 2017;33(11):3092-7.

44. Chaouachi A, Othman A Ben, Hammami R, Drinkwater EJ, Behm DG. The combination of plyometric and balance training improves sprint and shuttle run performances more often than plyometriconly training with children. J Strength Cond Res. 2014;28(2):401-12.

45. Hammami R, Granacher U, Makhlouf I, Behm DG, Chaouachi A. Sequencing effects of balance and plyometric training on physical performance in youth soccer athletes. J Strength Cond Res. 2016;30(12):3278-89.

46. Zghal F, Colson SS, Blain G, Behm DG, Granacher U, Chaouachi A. Combined resistance and plyometric training is more effective than plyometric training alone for improving physical fitness of pubertal soccer players. Front Physiol. 2019;7(10):1026-37.

47. Swain RA, Harsha DM, Baenziger J, Saywell RM. Do pseudoephedrine or phenylpropanolamine improve maximum oxygen uptake and time to exhaustion? Clin J Sport Med. 1997;7(3):168-73. 
48. Bogdanis GC, Tsoukos A, Kaloheri O, Terzis G, Veligekas P, Brown LE. Comparison between unilateral and bilateral plyometric training on single- and double-leg jumping performance and strength. J Strength Cond Res. $2019 ; 33(3): 633-40$.

49. Van Soest AJ, Roebroeck ME, Bobbert MF, Huijing PA, Schenau GJVI. A comparison of one-legged and two-legged countermovement jumps. Med Sci Sports Exerc. 1985;17(6):635-9.

50. Bobbert MF, De Graaf WW, Jonk JN, Casius LJR. Explanation of the bilateral deficit in human vertical squat jumping. J Appl Physiol. 2006;100(2):493-9.

51. Pappas E, Hagins M, Sheikhzadeh A, Nordin M, Rose D. Biomechanical differences between unilateral and bilateral landings from a jump: gender differences. Clin J Sport Med. 2007;17(4):263-8.

52. Altman DG, Royston P. The cost of dichotomising continuous variables. BMJ. $2006 ; 332: 1080$.

53. Sandercock GRH, Bromley PD, Brodie DA. Effects of exercise on heart rate variability: inferences from meta-analysis. Med Sci Sports Exerc. $2005 ; 37(3): 433-9$. 